\title{
Novel paradigms to measure variability of behavior in early childhood: posture, gaze, and pupil dilation
}

\author{
Robert Hepach ${ }^{1 *}$, Amrisha Vaish ${ }^{2}$ and Michael Tomasello ${ }^{1}$ \\ ${ }^{1}$ Department of Developmental and Comparative Psychology, Max Planck Institute for Evolutionary Anthropology, Leipzig, \\ Germany, ${ }^{2}$ Department of Psychology, University of Virginia, Charlottesville, VA, USA
}

OPEN ACCESS

Edited by:

Alia Martin,

Harvard University, USA

Reviewed by:

Gustaf Gredebäck

Uppsala University, Sweden

Emma L. Axelsson,

The Australian National University,

Australia

${ }^{*}$ Correspondence:

Robert Hepach

Department of Developmental and Comparative Psychology,

Max Planck Institute for Evolutionary Anthropology, Deutscher Platz 6,

Leipzig, Germany

hepach@eva.mpg.de

Specialty section

This article was submitted to

Developmental Psychology, a section of the journal

Frontiers in Psychology

Received: 07 January 2015 Accepted: 09 June 2015

Published: 09 July 2015

Citation:

Hepach R, Vaish A and Tomasello $M$ (2015) Novel paradigms to measure variability of behavior in early childhood: posture, gaze, and pupil

dilation.

Front. Psychol. 6:858. doi: 10.3389/fpsyg.2015.00858
A central challenge of investigating the underlying mechanisms of and the individual differences in young children's behavior is the measurement of the internal physiological mechanism and the involved expressive emotions. Here, we illustrate two paradigms that assess concurrent indicators of both children's social perception as well as their emotional expression. In one set of studies, children view situations while their eye movements are mapped onto a live scene. In these studies, children's internal arousal is measured via changes in their pupil dilation by using eye tracking technology. In another set of studies, we measured children's emotional expression via changes in their upperbody posture by using depth sensor imaging technology. Together, these paradigms can provide new insights into the internal mechanism and outward emotional expression involved in young children's behavior.

Keywords: posture, eye tracking, Kinect, pupillometry, pupil dilation, emotion, children, internal arousal

\section{Introduction}

Children's navigation through the social world rests on a set of socio-cognitive abilities that emerge during infancy and enable children to tune in to others' emotions and mental states (Carpenter et al., 1998; Tomasello et al., 2005; Grossmann and Johnson, 2007). These abilities in turn allow children to later establish and maintain social relationships. Nevertheless, there are individual differences with regards to the mechanisms that moderate children's social navigation and there is variability in children's responsiveness to others' feelings and desires (Dunn et al., 1991; Eisenberg et al., 1996; Rothbart et al., 2000; Knafo et al., 2008; Salley et al., 2013).

A central challenge to measuring the underlying processes of behavior is that these processes are often either internal or partially based on expressive emotions that occur briefly and rapidly in succession. However, recent advances in eye tracking and depth sensor imaging technology (1) allow us to 'listen in' on the internal states underlying behavior, and (2) provide a new lens through which emotional expressions become visible. Here, we illustrate two novel paradigms recently developed in our lab: one on children's responses to seeing others in need of help and another on children's postural changes following goal-oriented behavior. In our studies, the technology captures children's physiology and physiognomy from a distance so as to retain a natural setting in which the targeted behavior occurs.

The first paradigm was designed to capture children's gaze and pupil dilation as indices of attention and changes in internal arousal, respectively, in behavioral studies. Both variables are 
frequently assessed in studies on infants' physical and social cognition (Aslin and McMurray, 2004; Aslin, 2007; Falck-Ytter et al., 2013; Sirois and Brisson, 2014). However, while eye tracking has previously been employed to study cognition, our approach focuses on explaining children's behavior and addressing questions concerning its underlying motives and proximate mechanisms in active behavioral paradigms with children. Here we illustrate the use of this method to address questions regarding the specific motivations underlying children's prosocial behavior (see Gaze and Pupil Dilation). The second paradigm was designed to measure children's emotions, in particular their positive emotions as expressed in their posture. Previous research had focused on facial expressions and composite measures of gestures and posture to identify positive affect in young children through human coders' judgment. However, it has thus far not been possible to automatically capture children's emotions and to focus on specific body parts, e.g., the chest and hip. We apply this technique in situations where children achieve an outcome for themselves and we measure how their posture accordingly changes compared to a prior baseline (see Posture). This allows us to address questions regarding the emotions that accompany behavior, e.g., positive affect following success.

Here we illustrate the two paradigms, which involve capturing children's eye movements and pupil dilation to measure changes in autonomous nervous system (ANS) activity, and measuring children's posture in order to assess changes in their emotional state. We propose that these measures can not only provide insights into the internal mechanisms and emotional bases of children's behavior but also allow researchers to trace the physiological antecedents of children's actions to better understand the sources of variability and individual differences in social cognition and behavior.

\section{Gaze and Pupil Dilation}

For children to become competent social partners, they need to realize when others are in need of help, represent the appropriate solution, and have a sympathetic motivation to care for others' needs (Eisenberg and Miller, 1987; Zahn-Waxler et al., 1992; Dunfield, 2014; Warneken, 2015). The attention children pay to others' actions can be measured through tracking their eye movements and mapping them on a visual scene (Aslin and McMurray, 2004). Eye tracking is based on corneal reflection technology and provides numerous non-invasive indicators of attention, including fixations, saccades, anticipatory looking, and scan patterns (Aslin, 2007, 2012; Gredebäck et al., 2009; Oakes, 2012). This has opened up new ways of studying social cognition in infants and young children (Navab et al., 2012; Falck-Ytter et al., 2013; Tenenbaum et al., 2013). Both the time children spend looking at a scene and the pattern of eye movements can reveal the underlying structure of children's social attention (Falck-Ytter et al., 2006; Aslin, 2007; Frank et al., 2012; Fawcett and Gredebäck, 2013; Elsner et al., 2014).

An additional feature of modern corneal reflection eye trackers is the automatic capture of pupil diameter (Wang,
2011). Similar to other physiological measures such as heart rate or skin conductance, changes in pupil dilation reflect activation of the ANS (e.g., Kahneman et al., 1969; Libby et al., 1973; Bradley et al., 2008). This is particularly interesting for developmental research because whereas eye movements can reflect the distribution of attention, changes in pupil dilation may provide a measure of the degree of psychological involvement in pre-verbal and just-verbal populations (see Goldwater, 1972; Laeng et al., 2012; Sirois and Brisson, 2014, for reviews). Similar to the measure of children's eye movements, the measure of pupil dilation has also found application in infancy to study both physical and social cognition early in ontogeny (FalckYtter, 2008; Chatham et al., 2009; Jackson and Sirois, 2009; Gredebäck and Melinder, 2010, 2011; Geangu et al., 2011; Sirois and Jackson, 2012; Hepach and Westermann, 2013; Burkhouse et al., 2014).

The majority of previous work had implemented measures of gaze and pupil dilation in response to pictures or prerecorded video stimuli. To study more natural interactions researchers have adapted these set-ups for live paradigms wherein children sit facing an adult while the eye tracker records their eye movements and pupil size. Gredebäck et al. (2010) studied infants between the ages of 2-8 months in a live setting in which the experimenter or the mother sat facing the child. The authors found that infants' ability to follow others' gaze develops linearly and was more stable when facing a stranger compared to their mother (see Falck-Ytter et al., 2015, for a similar set-up). However, none of this previous work attempted to relate children's eye movements and pupil dilation to their behavior as a way to capture the mechanisms underlying children's behavior. Our aim was thus to extend the use of these measures in novel directions to study behavior more generally.

In one example we developed a paradigm to address questions regarding the motivation underlying young children's prosocial behavior. Specifically, we investigated how changes in internal arousal relate to children's own prosocial behavior. For this purpose we devised a behavioral helping paradigm within which we could capture children's gaze and pupil dilation to investigate the mechanisms underlying young children's helping behavior (Hepach et al., 2012). During the first 2 years of life children show a remarkable array of prosocial tendencies, including sharing with and comforting those in need of help (ZahnWaxler et al., 1992; Warneken and Tomasello, 2009; Svetlova et al., 2010; Dunfield, 2014; Eisenberg and Spinrad, 2014; Paulus, 2014; Warneken, 2015). However, much less is known about children's motives to help others. Therefore we inquired whether changes in young children's internal arousal reflect their motivation.

The general set-up and procedure of our studies is comparable to other developmental studies on children's prosocial behavior, yet we include a crucial difference. At pre-defined time points, children (24-month-olds) sit in front of an apparatus that resembles the facade of a house through which they can view the scene they themselves moments ago participated in. Children watch the scene on a computer screen, which shows a live video feed of the events on the 'other' side of the apparatus. Through a series of familiarizations (see also Troseth and DeLoache, 
1998), children learn that what they see on the screen is actually happening and that they can return to the task they were engaged in before they sat down (see Figure $\mathbf{1}$ for an illustration). In our studies, children view an adult carry out a task such as stacking cans until at one point the final object accidentally drops to the floor out of his/her reach. Children are then given the opportunity to subsequently help (see Hepach et al., 2012, for details).

While children sit in front of the apparatus, an eye tracker records both their eye movements and changes in pupil dilation (Tobii model X120, SMI models RED and RED-m). The live feed is presented on the computer screen by capturing a video from a USB webcam. The presentation software of the eye tracking system (Tobii Studio with Tobii systems and Experiment Center with SMI) allows both displaying a live video and simultaneously recording eye data at a frequency of at least $60 \mathrm{~Hz}$ and uses a standard calibration procedure to map children's gaze onto the computer screen (Gredebäck et al., 2009). It is furthermore possible to apply the same post-hoc gaze correction techniques suggested for eye tracking experiments (Frank et al., 2012). This allows for children's gaze to be mapped onto the live scene that they are observing and in turn provides a glimpse into the underlying process guiding their visual attention (see Figure 2). To further match children's pupillary responses to the live scene, several additional steps are necessary.

Assessing changes in children's internal arousal in active behavioral paradigms is particularly challenging given that pupil size variations are highly volatile in response to children's body movement during the study. We therefore further developed a technique (first described in detail in Hepach et al., 2012), which focuses on a specific component of pupil dilation rather than a mean over a specified time window. Changes in pupil diameter are a function of both sympathetic and parasympathetic nervous system activity. This results in the typical pupillary oscillations both dilating and constricting the pupil. Even in the dark, the pupils constantly oscillate (Wilhelm, 1991), making the signal of pupil diameter changes over time highly volatile. These oscillations are very different from smooth sine wave patterns given that the magnitude of the positive peaks (dilation) and negative peaks (constriction) as well as the time interval between peaks varies (Loewenfeld, 1993). Psycho-sensory stimulation will increase pupil dilation such that the peaks are higher than before the presentation of the stimulus. Analyses of changes in pupil diameter focus on robust indicators of psychologically induced effects such as the number of oscillations over several minutes (Warga et al., 2009), peak dilation (e.g., Laeng et al., 2012) or the amplitude of the pupillary light reflex (PLR; Steinhauer et al., 2000).

The PLR is the characteristic shape of the change in pupil size upon the presentation of light. With increasing stimulus luminance the pupils constrict. Loewenstein (1920) studied the influence of various emotional states in clinical patients and observed the PLR to be inhibited during induced stress, e.g., when subjects experienced tension or witnessed a startling event. The elicitation of a PLR by shining a light into subjects' eyes is part of the standard procedure in ophthalmology to assess parasympathetic and sympathetic nerves innervating both eyes (Wilhelm, 1991; see Bakes et al., 1990; Heller et al., 1990, for examples). Furthermore, several psychological stimuli inhibit the PLR, e.g., following negative emotional events (Bitsios et al., 2004) and with increased attention during task demands (Steinhauer et al., 2000). An increase in internal arousal results both in overall increased pupil dilation (Bradley et al., 2008) and in an inhibited PLR (Henderson et al., 2014; though see Nyström et al., 2015, for a different interpretation of the PLR in comparison to tonic pupil diameter). The advantage of measuring the PLR as an indicator of internal arousal is its quick assessment within 2-3 s. The crux is that the experimental manipulation has to occur before and not while the PLR is elicited.

During behavioral studies the presentation of visual stimuli on a computer screen causes the pupils to constrict to the luminance properties of an image. We have developed a technique in which we elicit two PLRs in brief succession, i.e., a colorful image flashes twice on the computer screen. The recorded data are exported to a text file and processed using software such as $\mathrm{R}$ or Matlab. The exported data need to be preprocessed to remove extreme values (see Hepach et al., 2012, 2013, for filter and interpolation examples). Subsequently, an algorithm identifies the two pupil minima in response to the colorful image and averages both values. The raw value of pupil diameter, i.e., the average minimum, is reflective of individual differences in children's arousal state. To further capture a change
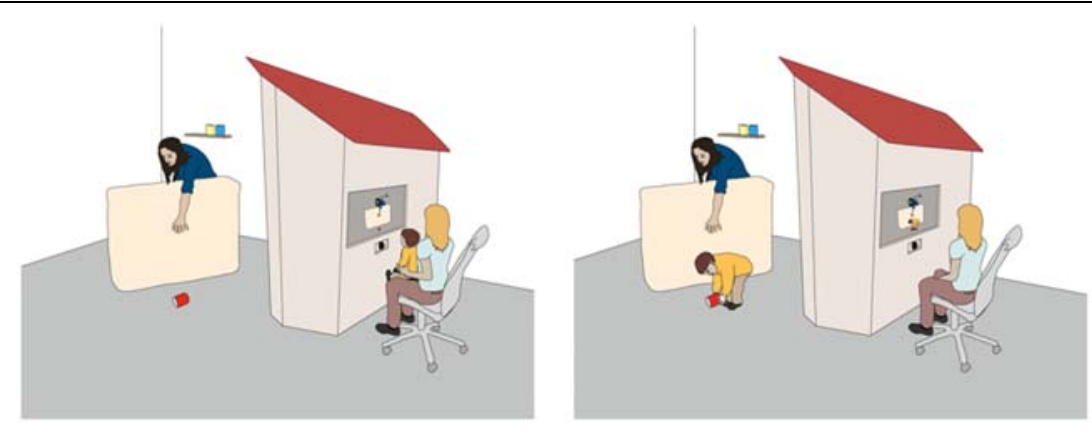

FIGURE 1 | The general set-up of the study. While children sit in front of the computer monitor, the eye tracking unit records both their eye movements and pupil diameter before they return to the adult's side. 


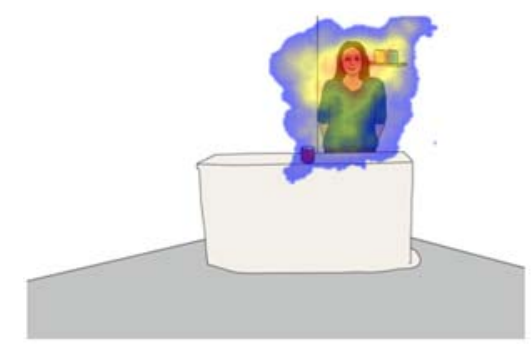

FIGURE 2 | Illustration of the scene children view while sitting in front of the apparatus. In the actual studies, children view a camera image of the adult carrying out her task. Since her behavior is never identical across participants, the illustrations here represent prototypical poses during specific phases of the study. The (left) represents the time during which the adult carried out her task, e.g., stacking cans, standing

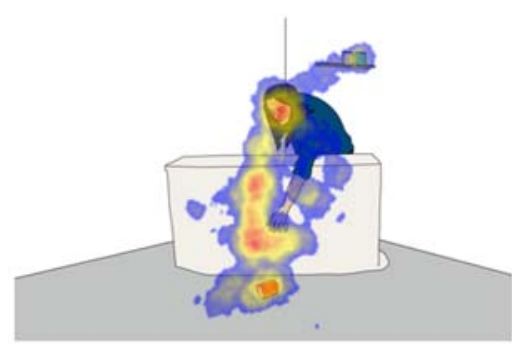

behind the table. The (right) represents the time the adult spent reaching for the dropped object. Each phase can last from a few seconds to several minutes. During that time, the eye tracker records children's gaze that can be mapped onto the illustrations to identify focal points of attention. The resulting focus maps reveal areas of low (blue) and high (red) attention. in children's internal arousal in response to an experimental manipulation, we present the measurement image both before (baseline measure) and after (process measure) the experimental manipulation (e.g., seeing an adult needing help). The change is measured as the percentage increase from baseline to process (see Figure 3).

Through recording children's eye movements as well as changes in pupil dilation in the domain of prosocial behavior, we have found that children's own internal arousal increases when others are not helped but decreases to an equal degree when help is provided either by children themselves or by others (Hepach et al., 2012). Crucially, the degree of children's internal arousal reflects individual differences in the latency with which they engage in helpful behavior, i.e., the greater children's pupil dilation is after witnessing the situation, the faster they are to subsequently help others (see Hepach et al., 2013).

While gaze and changes in pupil dilation reveal the processes that precede and underlie a behavior, they do not provide information regarding valence, i.e., whether children's responses are positive or negative in valence. For this purpose it is necessary to use an alternative measure of emotional expressiveness.

\section{Posture}

Children's attention to and involvement with others' needs can explain the motivations leading up to a behavior. An equally important aspect of motivation is the question of how a behavior is maintained and reinforced. In the following, we illustrate a novel paradigm to measure the sorts of positive emotions that follow from successful behavior. From as early as 2 years of age, children show noticeable postural changes following their successful attainment of a goal. Such emotional expressions provide a window to assess a subject's feelings, i.e., the internal state, especially if the emotion is studied in context (Lewis, 1997). Postural changes are accompanied by gestures such as pointing to the achievement or self-applauding (Heckhausen, 1987, 1988). By the age of 3 , children display an erect posture after succeeding on difficult tasks and conversely their posture decreases if they fail on easy ones (Lewis et al., 1992). Adults display similar changes in posture when they feel proud (Shiota et al., 2003; Tracy and Robins, 2004; Horberg et al., 2013), following athletic success (Weisfeld and Beresford, 1982), as a cue of social dominance (Schwartz et al., 1982), social status (Shariff and Tracy, 2009), and expertise (Martens and Tracy, 2013).

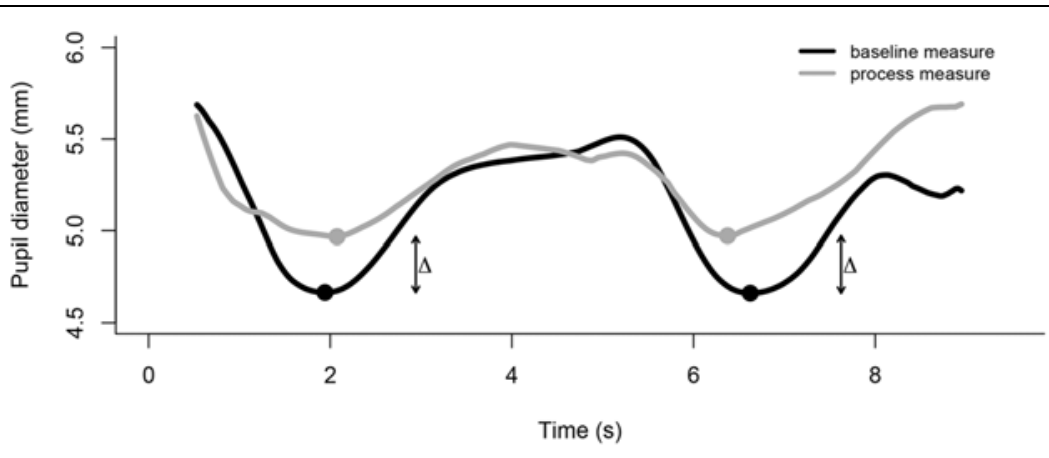

FIGURE 3 | The time course of the change in pupil size averaged across participants for two measurement time points, baseline and process. For each measurement point two pupillary light reflexes (PLRs) are elicited through presenting a bright stimulus on a dark background at 0 and
$5 \mathrm{~s}$, respectively. Upon presentation of the bright stimulus, the pupils constrict and re-dilate after reaching their respective minimum. An algorithm identifies the two minima and calculates the change from baseline to process pupil diameter. 
Postural changes are reliably identifiable from a person's gait (Montepare et al., 1987) as well as body movement (Dael et al., 2012) and the ability to detect pride from pictures emerges between 3 and 7 years of age (Tracy et al., 2005). The most common way to measure posture is to apply coding criteria to video recordings (e.g., Montepare et al., 1987; Heckhausen, 1988; Lewis et al., 1992; Shiota et al., 2003), photographs (e.g., Tracy and Robins, 2004; Shariff and Tracy, 2009; Martens and Tracy, 2013), drawings (Schwartz et al., 1982), and computer-animated mannequins or point light displays (Atkinson et al., 2004; Coulson, 2004). With these studies, then, there is a documented relation between success and the positive emotion expressed in an expanded upper-body posture. However, little research has assessed young children's postural changes in naturalistic situations without relying on the coding of additional cues such as clapping (e.g., Heckhausen, 1987). Here, we introduce a recently developed paradigm using depth sensor imaging technology to automatically capture individual differences and changes in children's posture.

\section{Automated Posture Assessment in Behavioral Paradigms}

To track participants' movement and the location of body points, we use a Mircosoft Kinect adapter in behavioral studies. The Kinect is a specialized camera that captures both an RGB-image, like any regular webcam, as well as the information of how far away each captured pixel is from the device itself. This depth sensor imaging is achieved through an emitted infrared light and a separate lens capturing the reflection of the projected light. If a person is within the device's tracking range $(\sim 1.5-4 \mathrm{~m}$ from the Kinect), the system estimates the $x, y$, and $z$-coordinates of up to 20 body points from the feet to the head (Shum et al., 2013; Stommel et al., 2015).

The Kinect allows for a relatively accurate and objective tracking of participants' body points and an assessment of body posture expansion (see Figure 4). The technology has been employed in several contexts studying infant-caregiver interactions (Nagai et al., 2012), children's behavior while playing cooperative games (Liu and LaFreniere, 2014), to assess and train motor abilities in clinical rehabilitation programs (Chang et al., 2013; de Greef et al., 2013; Luna-Oliva et al., 2013; Anzalone et al., 2014; Chung et al., 2014), and in interaction research (Won et al., 2014). In principal, the system can track multiple participants (Walczak et al., 2013) and it can be used to measure peripheral physiological measures such as respiratory rate (Burba et al., 2012). However, no experimental study has used the technology to capture the change in children's body posture as an indicator of emotional expression. This was our aim in recent work, which we describe next. Before doing so, we present the results from a study with adults in an attempt to validate the use of the technology in this way.

\section{Validation Study with Adults}

One of our central assumptions when using the Kinect technology in emotion research is that one can measure changes in upper-body posture that are related to positive and negative internal states. To test this assumption, we investigated whether the chest's center is more elevated when adult participants experience a positive emotion compared to a negative emotion.

\section{Participants}

Twelve naïve adult subjects (6 female, 14 years 5 months to 37 years 10 months, median age 26 years 4 months)
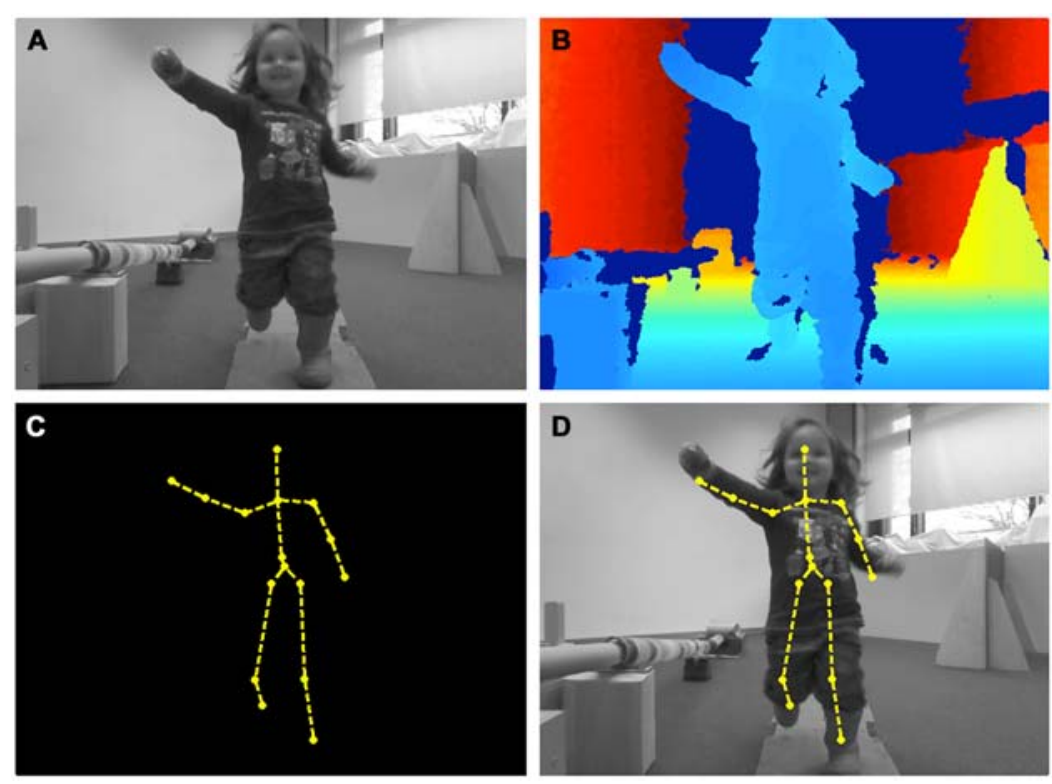

FIGURE 4 | The output of the Kinect motion sensor imaging technique. (A) RGB-channel, (B) depth contour image, (C) estimated skeletal joints, (D) mapping of the skeletal joints onto the RGB image. 
were recruited from the Max Planck Institute for Evolutionary Anthropology and gave informed consent prior to participating in the study.

\section{Materials and Design}

Each subject was asked to imagine experiencing four specific emotions, one at a time: joy, pride (positive emotions), disappointment, and guilt (negative emotions). We used a Kinect camera to record participants' body posture, i.e., the height of their chest's center as well as of their hip's center. Adults were presented with four test trials with one emotion each. The order was counterbalanced across participants.

\section{Procedure}

Before the emotion trials, participants were asked to walk toward the Kinect camera (height $=0.85 \mathrm{~m}$ from the ground, distance from participants' starting position $=3.7 \mathrm{~m}$, angle of the camera $=11^{\circ}$ ) such that a baseline assessment of the position of the chest's body joint could be made. The experimenter (blind to the study's hypotheses) read out the following instructions to participants: "This is a validation for a method to measure emotional expression. For this purpose we would like participants to walk toward the Kinect twice. At the very beginning we will conduct a baseline measurement for which we ask you to walk in a relaxed natural manner. Afterward I will read out the instructions for each emotion, four in total." At the beginning of each subsequent emotion trial the instructions were as follows (example joy): "The emotion to be displayed is joy. Can you recall an event that made you feel joy? Try to recall that feeling. Imagine the situation and surroundings. Take your time until you feel the emotion. Once you are ready give me a sign. Then you can walk toward the Kinect." Next, participants walked in the direction of the Kinect while the positions of both the chest's and hip's body point were recorded. For each trial (including baseline) we asked participants to walk toward the Kinect twice to average the data from both movements.

\section{Data Analysis}

The data were recorded with and analyzed in Matlab (see details below, in Section "Studies with Children"). Data from one trial of one participant (disappointment emotion) could not be used for analyses because of a system failure during recording. For each participant we calculated the change in the chest's height from baseline to the test trial for each of the four emotions and for each of 20 distance bins from the Kinect (1.2-3.2 $\mathrm{m}$ from the camera, $10 \mathrm{~cm}$ bin width). This controlled for differences in participants' walking speed. Furthermore, we averaged the values of joy and pride as well as disappointment and guilt to arrive at one composite positive and one composite negative emotion change score. For statistical analyses we further binned the data into four time windows of equal length and computed Wilcoxon exact paired tests with Bonferroni correction for multiple testing (adjusted significance level $p_{\text {adj }}=0.0125$ ). We carried out the identical analyses with children's hip point height to investigate whether the effects of emotions were specific to the upper-body posture.

\section{Results}

Adults' change in chest height from baseline was more elevated during the positive compared to the negative emotion events immediately after the emotion manipulation, $p=0.012$ (all other $p s>0.1$ ). This was not the case when performing the identical analyses on the hip's center (all $p$ s > 0.06; see Figure 5). These results suggested that adults' upper-body varies with the valence of the induced emotion. Measuring changes in upper body posture using the Kinect system can tap into the types of internal states involved in the experience and display during emotional episodes. This potentially makes the technology an interesting research tool to assess emotional expressions in young children.

\section{Studies with Children}

In our behavioral studies with 2-year-old children, participants can move around freely in a naturalistic setting without the need to attach point-light markers to their clothes. The Kinect 'draws' virtual points on participants' bodies. At specific time points during the study, the child moves toward the Kinect camera so that a full body image can be captured. In the following we provide data from one example (not reported with the original study) to illustrate that children's experience of an event that elicits a positive emotion reflects in changes of their body posture. At the beginning of the study we carried out a baseline measure during which children walked toward the Kinect without any experimental manipulation. At a later point in the study children manipulated a box to retrieve a toy that allowed them to continue with an attractive activity. Following this event, children again walked toward the Kinect camera. We hypothesized that experiencing this positive event would increase children's upper-body posture (see Figure 6 for an illustration).

The tracking of multiple body points allows one to isolate, for example, changes in shoulder and chest height from changes in hip height. That is, even though up to 20 body joints can be tracked with the Kinect, we focused on children's upper-body posture following work on signs of pride in adults (Montepare et al., 1987; Tracy and Robins, 2004). More specifically, we calculated the height of the chest's center as an indicator of postural expansion. Through assuming an upright posture, the shoulders are pushed back which in turn elevates the chest. In principle, a lowering of chest height could reflect a slumped posture, as documented in states of negative affect (Lewis et al., 1992).

The data were recorded running a script written in Matlab. At regular time intervals, the program records (1) information regarding the position of each body point in three-dimensional space, (2) a color image, (3) a depth image, as well as (4) the location of each point on the two-dimensional color image (see Figure 4). Separate analyses (written partly in Matlab and R) calculate the difference in chest height between the baseline phase and the measurement taken during the test trial after the experimental manipulation. This results in a baseline-corrected change score that indicates the change in upper-body posture (see Figure 7). 


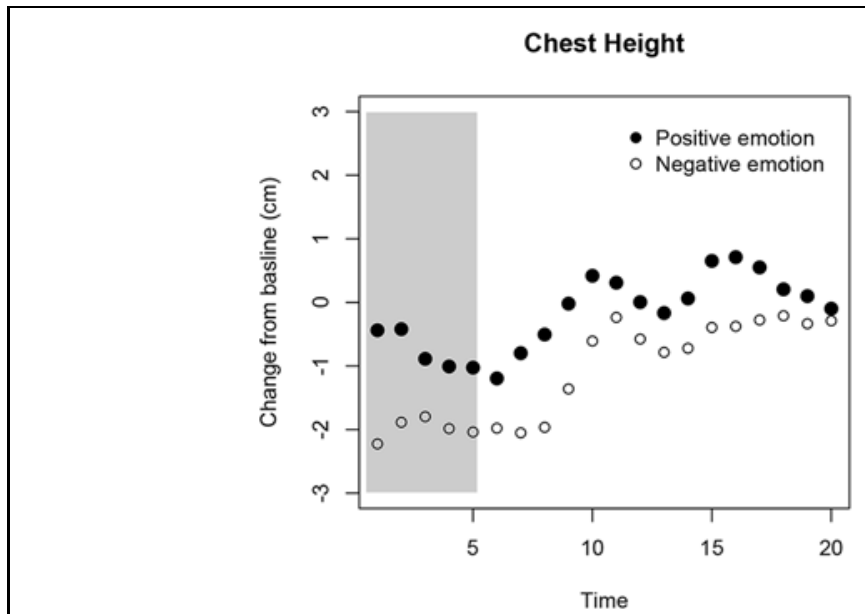

FIGURE 5|Results from the adult validation study. The $x$-axis represents the time after the emotion was elicited and as adults walked toward the Kinect. The $y$-axis shows the relative change in height for participants' chest (left) and hip (right). At each time point

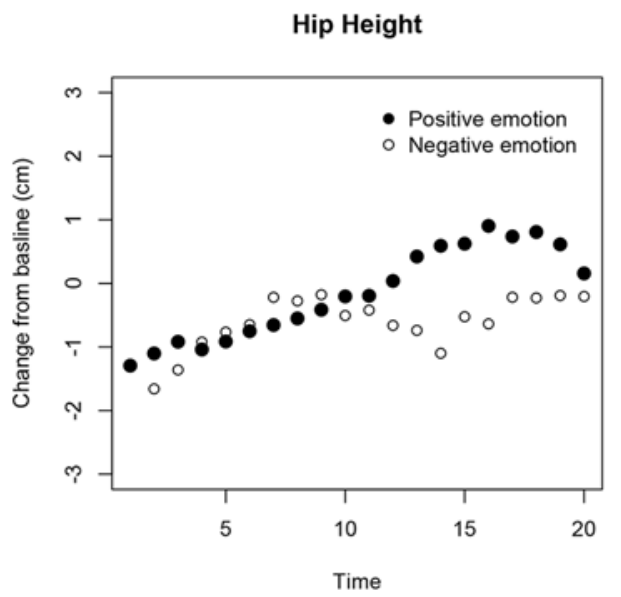

the median for the two positive and the two negative emotions is plotted. The gray area marks the time window where the difference between the two types of emotions was statistically significant (corrected for multiple testing).
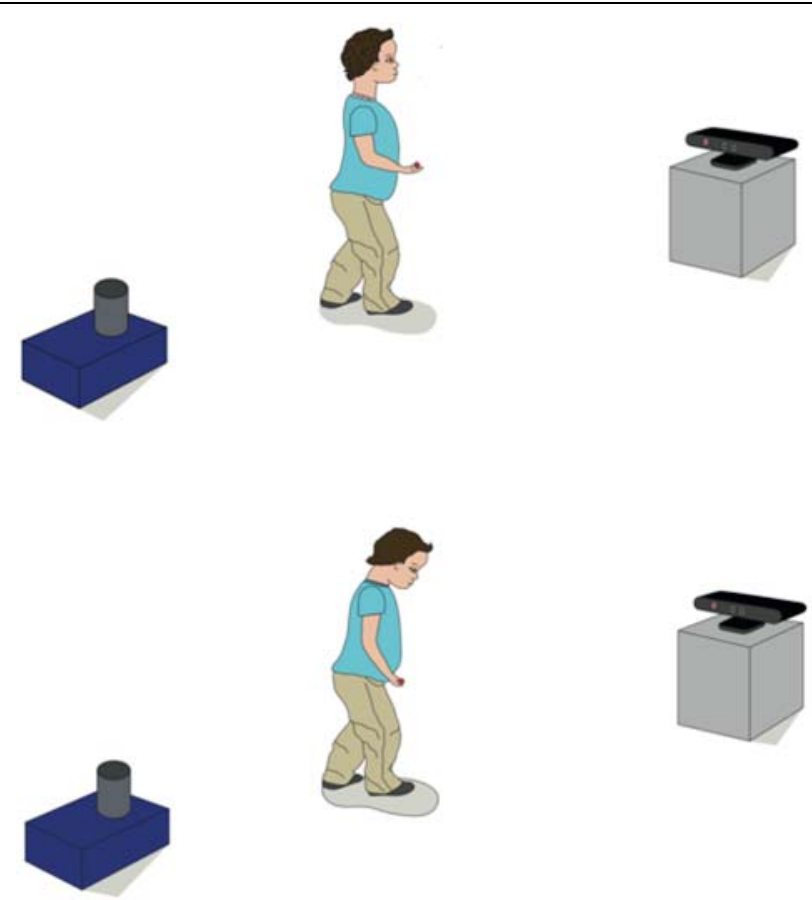

FIGURE 6 | The experimental setup of the study. Children walked toward the Kinect camera after they either experienced a positive (top) or neutral event (bottom). During the positive event children retrieved an object from the blue box that allowed them to continue an activity while in the neutral event no experimental manipulation occurred.

\section{Coding of Children's Affect}

The assumption underlying the interpretation of the Kinect data is that changes in children's chest but not hip height reflect changes in positive affect. The more positive children feel, the more their upper-body posture should expand. To investigate this

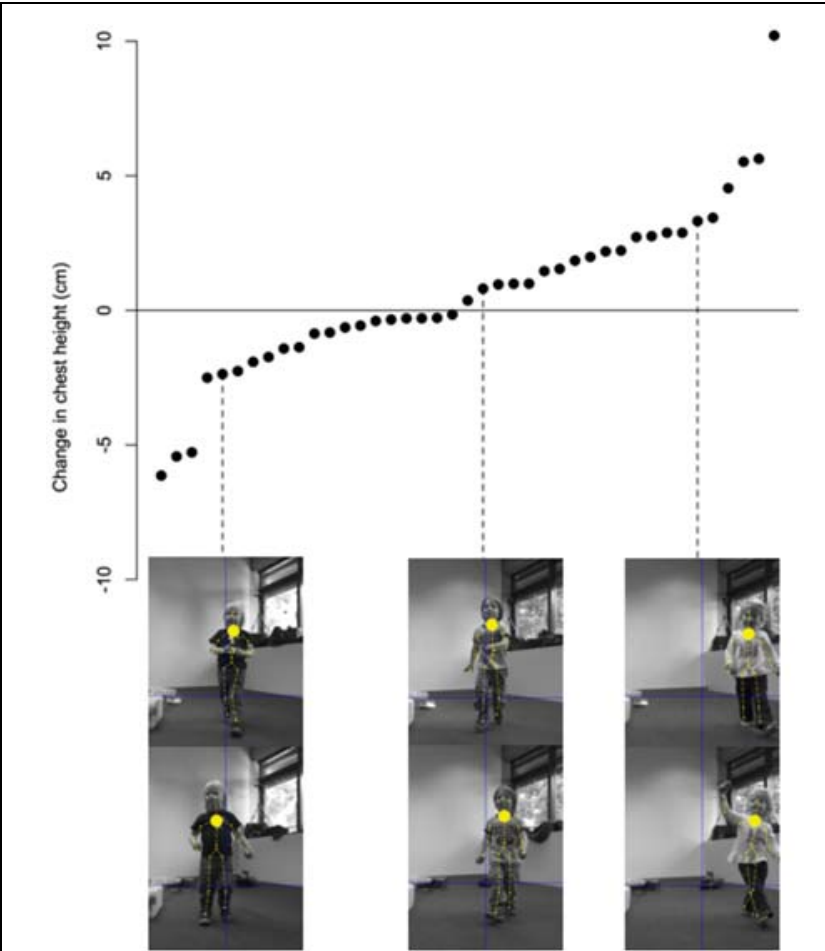

FIGURE 7 | An illustration of the data collected by the Kinect camera. Each data point represents the change in the height of the chest's center for each participant. The values are sorted from the lowest change to the largest change in height. For three participants illustrations are provided from the baseline sequence (top row) and from the later taken process sequence (bottom row).

relation we asked two adults (blind to the study's hypotheses and type of trial) to rate the recordings of children's behavior along several dimensions. 
The material consisted of the recordings of 48 children (25 girls, age range 29 months; 4 days to 31 months; 5 days; median age 30 months; 16 days) with two trials per child (baseline and test). The Kinect system could not record data for seven children on either trial or on both. For each trial coders were provided with the picture frames for the sequence when children started walking toward the Kinect, i.e., the exact same frames that were used for the automated posture analysis. The picture frames did not depict the skeletal information provided by the Kinect system.

For each trial the two coders were given the following instructions along with the SAM (self-assessment-manikin) rating scale (Bradley and Lang, 1994): "The SAM-rating consists of a valence coding and an arousal coding. The scale ranges from 9 to 1 . For each trial, please answer the following questions: How pleasant is the emotion that the child is experiencing (very pleasant $\sim 9$, very unpleasant $\sim 1$ ) ? How arousing is the emotion that the child is experiencing (very arousing $\sim 9$, not at all arousing $\sim 1)$ ?" In addition we asked coders what emotion they saw the child displaying and what features they paid attention to when identifying the emotion. The aim of the latter question was to investigate which emotions coders spontaneously associate with the behavior of the child. The ratings of the two coders were positively correlated, both with regards to rating valence $\left[\rho_{\text {spearman }}(n=90)=0.74\right.$, $p<0.001, \mathrm{ICC}=0.63]$ and arousal $\left[\rho_{\text {spearman }}(n=90)=0.42\right.$, $p<0.001$, ICC $=0.36$ ]. We therefore averaged both codings to arrive at composite measures of both valence and arousal.

The results showed that the rated pleasantness of the children's affect was greater in the test $(M=6.45, \mathrm{SD}=1.66)$ compared to the baseline $(M=5.83, \mathrm{SD}=1.73)$ trial, $t(41)=2.24, p=0.031$. On the other hand, there was no difference in the ratings of children's arousal between the baseline and test trial, $t(41)=1.17$, $p=0.25$ (see Figure 8). This suggests that the experimental manipulation of attaining a goal for oneself makes children appear to experience more pleasure compared to a baseline level. With regards to the coders' ratings of children's affect during the test trial and the change in children's posture, there was no overall relation between the two variables, $\rho(n=41)=0.087$, $p=0.59$. However, very few trials were coded as 'negative,' i.e., with a value of less than $5(17 \%)$. When focusing the analyses on the positive affect realm, i.e., ratings from 5 to 9 , the degree of children's experienced affect was positively related to the change in their chest height from the baseline to the test trial. Children with ratings of high positive affect also tended to show a greater increase in upper-body posture, $\rho(n=34)=0.37, p=0.03$ (see Figure 8). On the other hand there was no such relation with respect to children's lower-body posture, i.e., the change of hip height, $\rho(n=34)=0.08, p=0.67$ (see Figure 8). In addition, no statistically significant relations emerged between children's rated degree of arousal and the change in their chest or hip height, $p s>0.09$. Furthermore, the most frequently rated emotion after the experimental manipulation was 'happy' (see Table 1 for details) and the most frequent features that coders paid attention to were children's smile, posture, and gait (see Table 2 for details).

Overall, the Kinect depth sensor imaging technology not only provides information on individual differences in overall body size but also registers subtle changes in posture. Given the link between positive affect and increased body posture, the Kinect is an extremely useful new tool to measure emotional expressions and motivational states in children.

\section{Summary and Future Directions}

The paradigms described in this brief overview aim to capture the underlying mechanism of behavior and the types of expressive emotions that follow from it. Children's eye movements in response to live events in behavioral paradigms reveal how they allocate their attention. Likewise, changes in their pupil

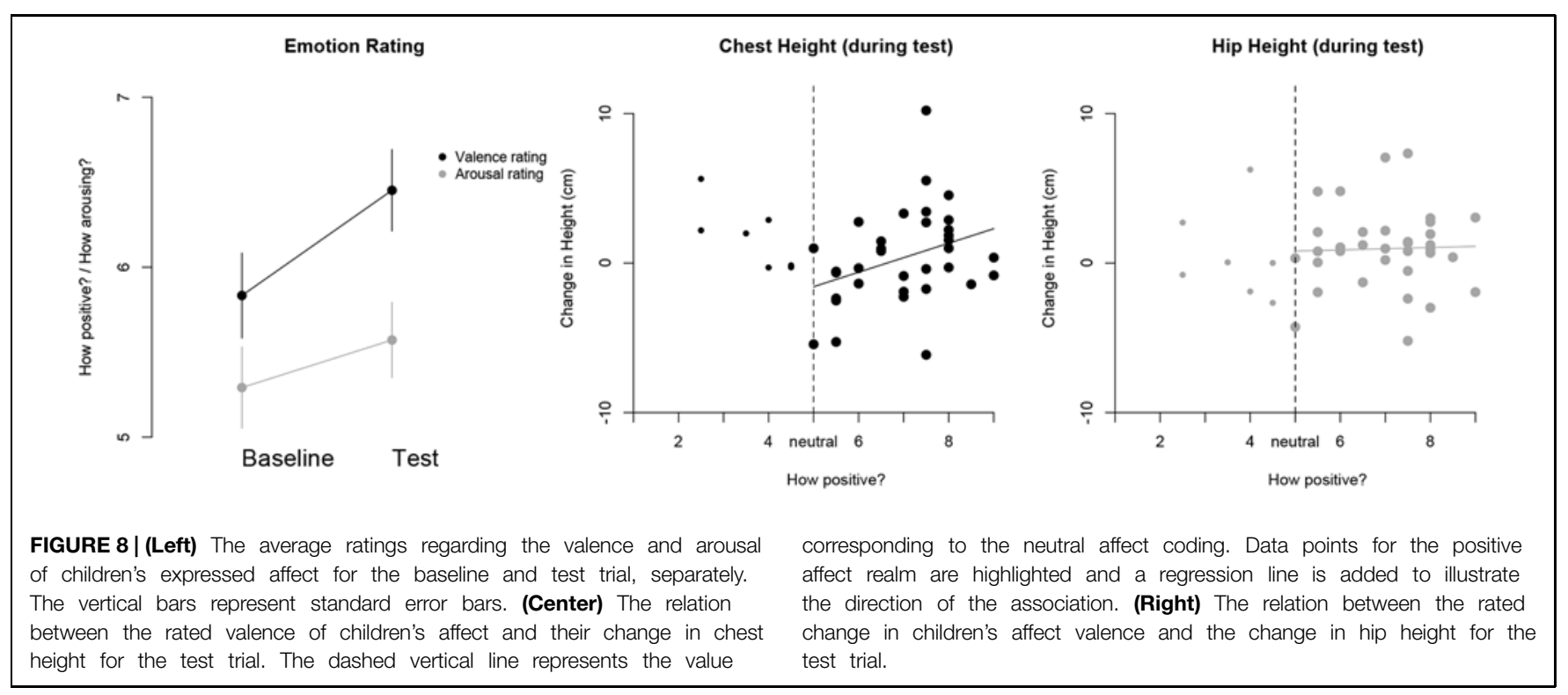


TABLE 1 | The frequency of the type of emotion children expressed during the test trial as identified by the two coders.

\begin{tabular}{|c|c|c|c|c|c|c|c|c|c|c|}
\hline & Angry & Sad & Embarrassed /uncertain & Disappointed & Disinterested & Surprise & Neutral & Active & Proud & Happy \\
\hline Frequency & 1 & 2 & 23 & 1 & 6 & 3 & 4 & 6 & 14 & 52 \\
\hline
\end{tabular}

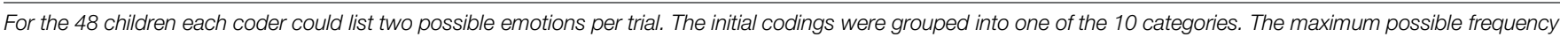
for an emotion was 96, i.e., both coders identified the emotion for each of the 48 trials.

TABLE 2 | The features of children's behavior during the test trial that coders referred to when making the decision of what emotion the child was expressing.

\begin{tabular}{cccccc}
\hline & Smile & $\begin{array}{l}\text { Other facial } \\
\text { feature }\end{array}$ & $\begin{array}{l}\text { Looking } \\
\text { direction }\end{array}$ & Posture & Gait \\
\hline Frequency & 43 & 3 & 6 & 41 & 49
\end{tabular}

Each coder could provide two features per trial. The initial codings were grouped into one of the five categories. The maximum possible frequency for a feature was 96, i.e., both coders referred to this feature for each of the 48 trials.

dilation indicate the strength of their motivation. Individual differences in children's internal arousal before they carry out an action are related to how quickly they do so. In addition to these measures of internal arousal, changes in upper-body posture reflect children's positive emotional state after carrying out an action. A more straight and upright posture is indicative of a positive emotion while a hanging posture may reflect a negative emotional state (see also Lewis et al., 1992). These methods allow researchers to address novel questions regarding the underlying mechanisms of behavior (using pupil dilation) as well as children's emotional expressions that accompany behavior (using depth sensor imaging). One direction for future studies using eye tracking systems is to collect gaze and pupil data without the need for children to look at a computer screen. The fact that children have to move out of the live situation and temporarily sit in front of a separate apparatus interrupts the task they are involved in. In particular, younger children have difficulty sitting on their parent's lap after an engaging activity. This can result in inattentiveness and fewer chances to gather data points. Moreover, the image on the screen is only a 2-dimensional representation of a 3-dimensional space. In principle, both Tobii and SMI eye tracking systems can map participants' gaze onto a 'real' scene. This is a direction for future research to explore, especially with the emergent use of head-mounted cameras and eye tracking systems (Aslin, 2009; Smith et al., 2014). With regard to using the Kinect camera, an interesting further step is to explore whether the system can also capture other emotions, including those with negative affect such as shame and guilt. In particular, investigating the relation between the various body points, e.g., head vs. shoulders, will provide an interesting avenue for future research given that we have thus far only explored the change in chest height. In this way the Kinect could not only be used to address questions regarding the positive emotions that follow from successful actions but also to study emotional expression in children more broadly.

In the examples given here, measures of pupil dilation assessed children's internal arousal before they carried out an action while measures of posture were taken after children completed their action. In principle, neither technology needs to be restricted to these uses. In fact, there is now work measuring changes in internal arousal both before and after an experimental manipulation to investigate different motivations underlying children's helping behavior (Hepach et al., 2012). Likewise, children's emotional expression in their body posture may already change in anticipation of a positive or negative event.

The study of individual differences in children's behavior relies in part on providing novel dependent measures with which to investigate subtle differences in behavior. In particular, studying the underlying mechanisms allows researchers to address questions that go beyond asking whether or not a behavior occurred in a given context. In the present paper we have provided the example of children's prosocial behavior. Children, much like adults, do not help all the time and understanding the motivations that facilitate or inhibit helping is critical in our understanding of human prosociality. Changes in children's internal arousal, as measured via variation in pupil dilation, do not only reveal how children respond to others in need but also systematically relate to their willingness to engage in helping. Children with greater pupil dilation in response to seeing a person in need are faster to subsequently help (Hepach et al., 2013). In more recent work we have found that children show greater pupil dilation when viewing an adult struggling with an instrumental task compared to a non-social case that portrays an instrumental problem without a person present (Hepach et al., in review). In that study we further specified that it is children's process- but not baseline-measure that systematically relates to individual differences in helping behavior. Similar to the findings by Hepach et al. (2013), children with greater pupil dilation in response to seeing an adult in need were faster to help. Future work will have to investigate whether this relation between internal arousal and the latency to carry out a behavior is specific to children's helping or whether it applies to other contexts as well (such as play).

In addition to understanding the underlying mechanisms of behavior it is equally important to study variability in the expressive emotions that accompany children's behavior. Emotions can be measured via various modalities such as the voice or face. In the present paper we have illustrated a novel paradigm to measure children's posture after achieving a positive outcome. Such changes are relevant to behavior given that children are more likely to carry out an action if they find it enjoyable. Children did not only show variability in their emotional expression (see Figure 7) but the change in their upper-body posture systematically related to adult coders' ratings of the valence of children's expressed emotion. Children with greater increase in posture were rated to feel more positive. One possible avenue for future research is to investigate how others 
perceive and respond to children's postural changes which may in turn have an impact not only on how children's subsequent posture changes but also on how they experience the actual emotion underlying the postural change (see also Carney et al., 2010).

Early in ontogeny, the technologies described here can provide a window into the underlying mechanisms of behavior. This is particularly relevant given that different processes can result in the same behavior (Karmiloff-Smith et al., 2014). The two examples in the present paper show that variability in children's behavior is meaningful, e.g., children's helping behavior is related to changes in their internal arousal. While the implementation of both pupillometry and depth sensor imaging was here illustrated in two specific contexts, their application need not be limited to children's prosocial and goal-oriented behavior. In fact, any researcher interested in variability of behavior early in ontogeny may find the research tools illustrated here useful for various forms of behavior in different contexts. Internal measures of attention and arousal as well as measures of

\section{References}

Anzalone, S. M., Tilmont, E., Boucenna, S., Xavier, J., Jouen, A. L., Bodeau, N., et al. (2014). How children with autism spectrum disorder behave and explore the 4-dimensional (spatial 3D++ time) environment during a joint attention induction task with a robot. Res. Autism Spectr. Disord. 8, 814-826. doi: 10.1016/j.rasd.2014.03.002

Aslin, R. N. (2007). What's in a look? Dev. Sci. 10, 48-53. doi: 10.1111/j.14677687.2007.00563.x

Aslin, R. N. (2009). How infants view natural scenes gathered from a head-mounted camera. Optom. Vis. Sci. 86, 561-565. doi: 10.1097/OPX.0b013e3181a76e96

Aslin, R. N. (2012). Infant eyes: a window on cognitive development. Infancy 17, 126-140. doi: 10.1111/j.1532-7078.2011.00097.x

Aslin, R. N., and McMurray, B. (2004). Automated corneal-reflection eye tracking in infancy: methodological developments and applications to cognition. Infancy 6, 155-163. doi: 10.1207/s15327078in0602_1

Atkinson, A. P., Dittrich, W. H., Gemmell, A. J., and Young, A. W. (2004). Emotion perception from dynamic and static body expressions in point-light and full-light displays. Perception 33, 717-746. doi: 10.1068/ p5096

Bakes, A., Bradshaw, C. M., and Szabadi, E. (1990). Attenuation of the pupillary light reflex in anxious patients. Br. J. Clin. Pharmacol. 30, 377-381. doi: 10.1111/j.1365-2125.1990.tb03787.x

Bitsios, P., Szabadi, E., and Bradshaw, C. M. (2004). The fear-inhibited light reflex: importance of the anticipation of an aversive event. Int. J. Psychophysiol. 52, 87-95. doi: 10.1016/j.ijpsycho.2003.12.006

Bradley, M. M., and Lang, P. J. (1994). Measuring emotion: the self-assessment manikin and the semantic differential. J. Behav. Ther. Exp. Psychiatry 25, 49-59. doi: 10.1016/0005-7916(94)90063-9

Bradley, M. M., Miccoli, L., Escrig, M. A., and Lang, P. J. (2008). The pupil as a measure of emotional arousal and autonomic activation. Psychophysiology 45, 602-607. doi: 10.1111/j.1469-8986.2008.00654.x

Burba, N., Bolas, M., Krum, D. M., and Suma, E. A. (2012). "Unobtrusive measurement of subtle nonverbal behaviors with the Microsoft Kinect," in Proceedings of the 2012 IEEE Virtual Reality Short Papers and Posters (Washington, DC: IEEE Computer Society), 1-4. doi: 10.1109/vr.2012.6180952

Burkhouse, K. L., Siegle, G. J., and Gibb, B. E. (2014). Pupillary reactivity to emotional stimuli in children of depressed and anxious mothers. J. Child Psychol. Psychiatry 55, 1009-1016. doi: 10.1111/jcpp.12225

Carney, D. R., Cuddy, A. J., and Yap, A. J. (2010). Power posing brief nonverbal displays affect neuroendocrine levels and risk tolerance. Psychol. Sci. 21, 13631368. doi: $10.1177 / 0956797610383437$ emotional expressiveness move researchers closer to the source of variability. Together, these measures can be considered additions to the scientific toolbox with which researchers study the origins and development of children's social cognition and behavior. It will be a central challenge for future work to implement these techniques to study age-related changes in children's social cognitive development.

\section{Acknowledgments}

We thank Isabelle de Gaillande-Mustoe, Chrsitian Skupin, and Georg Keller for their help with data collection, Johannes Liebold for his help with piloting the adult validation study procedure, Marike Schreiber and Cristina Zickert for providing the illustrations, and Bahar Köymen for helpful discussions during preparation of the manuscript. We thank Robert Schettler for IT and Matlab support as well as Marco Roggero for his help with Matlab.

Carpenter, M., Nagell, K., and Tomasello, M. (1998). Social cognition, joint attention, and communicative competence from 9 to 15 months of age. Monogr. Soc. Res. Child Dev. 63, 1-174.

Chang, Y. J., Han, W. Y., and Tsai, Y. C. (2013). A Kinect-based upper limb rehabilitation system to assist people with cerebral palsy. Res. Dev. Disabil. 34, 3654-3659. doi: 10.1016/j.ridd.2013.08.021

Chatham, C. H., Frank, M. J., and Munakata, Y. (2009). Pupillometric and behavioral markers of a developmental shift in the temporal dynamics of cognitive control. Proc. Natl. Acad. Sci. U.S.A. 106, 5529-5533. doi: 10.1073/pnas.0810002106

Chung, I. C., Huang, C. Y., Yeh, S. C., Chiang, W. C., and Tseng, M. H. (2014). "Developing kinect games integrated with virtual reality on activities of daily living for children with developmental delay," in Advanced Technologies, Embedded and Multimedia for Human-Centric Computing, eds Y.-M. Huang, H.-C. Chao, D.-J. Deng, and J. J. J. H. Park (Dordrecht: Springer Netherlands), 1091-1097.

Coulson, M. (2004). Attributing emotion to static body postures: recognition accuracy, confusions, and viewpoint dependence. J. Nonverbal Behav. 28, 117139. doi: 10.1023/B:JONB.0000023655.25550.be

Dael, N., Mortillaro, M., and Scherer, K. R. (2012). Emotion expression in body action and posture. Emotion 12, 1085-1101. doi: 10.1037/a0025737

de Greef, K., van der Spek, E. D., and Bekker, T. (2013). “Designing kinect games to trainmotor skills for mixed ability players," in Games for Health: Proceedings of the 3rd European Conference on Gaming and Playful Interaction in Health Care, eds B. Schouten, S. Fedtke, T. Bekker, M. Schijven, and A. Gekker (Wiesbaden: Springer Fachmedien), 197-205.

Dunfield, K. A. (2014). A construct divided: prosocial behavior as helping, sharing, and comforting subtypes. Front. Psychol. 5:958. doi: 10.3389/fpsyg.2014.00958

Dunn, J., Brown, J., Slomkowski, C., Tesla, C., and Youngblade, L. (1991). Young children's understanding of other people's feelings and beliefs: individual differences and their antecedents. Child Dev. 62, 1352-1366. doi: $10.2307 / 1130811$

Eisenberg, N., Fabes, R. A., Murphy, B., Karbon, M., Smith, M., and Maszk, P. (1996). The relations of children's dispositional empathy-related responding to their emotionality, regulation, and social functioning. Dev. Psychol. 32, 195-209. doi: 10.1037/0012-1649.32.2.195

Eisenberg, N., and Miller, P. A. (1987). The relation of empathy to prosocial and related behaviors. Psychol. Bull. 101, 91-119. doi: 10.1037/0033-2909. 101.1.91

Eisenberg, N., and Spinrad, T. L. (2014). "Multidimensionality of prosocial behavior," in Prosocial Development: A Multidimensional Approach, eds L. M. Padilla-Walker and G. Carlo (Oxford: Oxford University Press), 17-39. doi: 10.1093/acprof:oso/9780199964772.003.0002 
Elsner, C., Bakker, M., Rohlfing, K., and Gredebäck, G. (2014). Infants' online perception of give-and-take interactions. J. Exp. Child Psychol. 126, 280-294. doi: 10.1016/j.jecp.2014.05.007

Falck-Ytter, T. (2008). Face inversion effects in autism: a combined looking time and pupillometric study. Autism Res. 1, 297-306. doi: 10.1002/aur.45

Falck-Ytter, T., Bölte, S., and Gredebäck, G. (2013). Eye tracking in early autism research. J. Neurodev. Disord. 5, 28. doi: 10.1186/1866-1955-5-28

Falck-Ytter, T., Carlström, C., and Johansson, M. (2015). Eye contact modulates cognitive processing differently in children with autism. Child Dev. 86, 37-47. doi: $10.1111 /$ cdev.12273

Falck-Ytter, T., Gredebäck, G., and von Hofsten, C. (2006). Infants predict other people's action goals. Nat. Neurosci. 9, 878-879. doi: 10.1038/nn1729

Fawcett, C., and Gredebäck, G. (2013). Infants use social context to bind actions into a collaborative sequence. Dev. Sci. 16, 841-849. doi: 10.1111/desc. 12074

Frank, M. C., Vul, E., and Saxe, R. (2012). Measuring the development of social attention using free-viewing. Infancy 17, 355-375. doi: $10.1111 /$ j.15327078.2011.00086.x

Geangu, E., Hauf, P., Bhardwaj, R., and Bentz, W. (2011). Infant pupil diameter changes in response to others' positive and negative emotions. PLoS ONE 6:e27132. doi: 10.1371/journal.pone.0027132

Goldwater, B. C. (1972). Psychological significance of pupillary movements. Psychol. Bull. 77, 340-355. doi: 10.1037/h0032456

Gredebäck, G., Fikke, L., and Melinder, A. (2010). The development of joint visual attention: a longitudinal study of gaze following during interactions with mothers and strangers. Dev. Sci. 13, 839-848. doi: 10.1111/j.14677687.2009.00945.x

Gredebäck, G., Johnson, S., and von Hofsten, C. (2009). Eye tracking in infancy research. Dev. Neuropsychol. 35, 1-19. doi: 10.1080/87565640903325758

Gredebäck, G., and Melinder, A. (2010). Infants' understanding of everyday social interactions: a dual process account. Cognition 114, 197-206. doi: 10.1016/j.cognition.2009.09.004

Gredebäck, G., and Melinder, A. (2011). Teleological reasoning in 4-month-old infants: pupil dilations and contextual constraints. PLoS ONE 6:e26487. doi: 10.1371/journal.pone.0026487

Grossmann, T., and Johnson, M. H. (2007). The development of the social brain in human infancy. Eur. J. Neurosci. 25, 909-919. doi: 10.1111/j.14609568.2007.05379.x

Heckhausen, H. (1987). "Emotional components of action: their ontogeny as reflected in achievement behavior," in Curiosity, Imagination and Play: On the Development of Spontaneous Cognitive and Motivational Processes, eds D. Görlitz and J. F. Wohlwill (Hillsdale, NJ: Lawrence Erlbaum), 326-348.

Heckhausen, J. (1988). Becoming aware of one's competence in the second year: developmental progression within the mother child dyad. Int. J. Behav. Dev. 11, 305-326. doi: 10.1177/016502548801100302

Heller, P. H., Perry, F., Jewett, D. L., and Levine, J. D. (1990). Autonomic components of the human pupillary light reflex. Invest. Ophthalmol. Vis. Sci. $31,156-162$.

Henderson, R. R., Bradley, M. M., and Lang, P. J. (2014). Modulation of the initial light reflex during affective picture viewing. Psychophysiology 51, 815-818. doi: 10.1111/psyp. 12236

Hepach, R., Vaish, A., and Tomasello, M. (2012). Young children are intrinsically motivated to see others helped. Psychol. Sci. 23, 967-972. doi: 10.1177/0956797612440571

Hepach, R., Vaish, A., and Tomasello, M. (2013). A new look at children's prosocial motivation. Infancy 18, 67-90. doi: 10.1111/j.1532-7078.2012.00130.x

Hepach, R., and Westermann, G. (2013). Infants' sensitivity to the congruence of others' emotions and actions. J. Exp. Child Psychol. 115, 16-29. doi: 10.1016/j.jecp.2012.12.013

Horberg, E. J., Kraus, M. W., and Keltner, D. (2013). Pride displays communicate self-interest and support for meritocracy. J. Pers. Soc. Psychol. 105, 24-37. doi: $10.1037 / \mathrm{a} 0032849$

Jackson, I., and Sirois, S. (2009). Infant cognition: going full factorial with pupil dilation. Dev. Sci. 12, 670-679. doi: 10.1111/j.1467-7687.2008.00805.x

Kahneman, D., Tursky, B., Shapiro, D., and Crider, A. (1969). Pupillary, heart rate, and skin resistance changes during a mental task. J. Exp. Psychol. 79, 164-167. doi: 10.1037/h0026952

Karmiloff-Smith, A., Casey, B. J., Massand, E., Tomalski, P., and Thomas, M. S. (2014). Environmental and genetic influences on neurocognitive development the importance of multiple methodologies and time-dependent intervention. Clin. Psychol. Sci. 2, 628-637. doi: 10.1177/2167702614521188

Knafo, A., Zahn-Waxler, C., Van Hulle, C., Robinson, J. L., and Rhee, S. H. (2008). The developmental origins of a disposition toward empathy: genetic and environmental contributions. Emotion 8, 737-752. doi: 10.1037/a00 14179

Laeng, B., Sirois, S., and Gredebäck, G. (2012). Pupillometry a window to the preconscious? Perspect. Psychol. Sci. 7, 18-27. doi: 10.1177/1745691611427305

Lewis, M. (1997). The self in self-conscious emotions. Ann. N. Y. Acad. Sci. 818, 119-142. doi: 10.1111/j.1749-6632.1997.tb48251.x

Lewis, M., Alessandri, S. M., and Sullivan, M. W. (1992). Differences in shame and pride as a function of children's gender and task difficulty. Child Dev. 63, 630-638. doi: 10.2307/1131351

Libby, W. L., Lacey, B. C., and Lacey, J. I. (1973). Pupillary and cardiac activity during visual attention. Psychophysiology 10, 270-294. doi: 10.1111/j.14698986.1973.tb00526.x

Liu, C., and LaFreniere, P. (2014). The effects of age-mixing on peer cooperation and competition. Hum. Ethol. Bull. 29, 4-17.

Loewenfeld, I. E. (1993). The Pupil: Anatomy, Physiology, and Clinical Applications. Detroit, MI: Wayne State University Press.

Loewenstein, O. (1920). Experimentelle beitrage zur lehre von den katatonischen pupillenveranderungen. Monatschr. Psychiatr. Neurol. 47, 194-215. doi: $10.1159 / 000190690$

Luna-Oliva, L., Ortiz-Gutiérrez, R. M., Cano-de la Cuerda, R., Piédrola, R. M., Alguacil-Diego, I. M., Sánchez-Camarero, C., et al. (2013). Kinect Xbox 360 as a therapeutic modality for children with cerebral palsy in a school environment: a preliminary study. NeuroRehabilitation 33, 513-521. doi: 10.3233/ NRE-131001

Martens, J. P., and Tracy, J. L. (2013). The emotional origins of a social learning bias does the pride expression cue copying? Soc. Psychol. Pers. Sci. 4, 492-499. doi: 10.1177/1948550612457958

Montepare, J. M., Goldstein, S. B., and Clausen, A. (1987). The identification of emotions from gait information. J. Nonverbal Behav. 11, 33-42. doi: 10.1007/BF00999605

Nagai, Y., Nakatani, A., Qin, S., Fukuyama, H., Myowa-Yamakoshi, M., and Asada, M. (2012). "Co-development of information transfer within and between infant and caregiver," in Proceedings of the 2012 IEEE International Conference on Development and Learning and Epigenetic Robotics (ICDL), San Diego, CA: IEEE, 1-6. doi: 10.1109/DevLrn.2012.6400879

Navab, A., Gillespie-Lynch, K., Johnson, S. P., Sigman, M., and Hutman, T. (2012). Eye-tracking as a measure of responsiveness to joint attention in infants at risk for autism. Infancy 17, 416-431. doi: 10.1111/j.1532-7078.2011.00082.x

Nyström, P., Gredebäck, G., Bölte, S., and Falck-Ytter, T. (2015). Hypersensitive pupillary light reflex in infants at risk for autism. Mol. Autism 6, 10. doi: 10.1186/s13229-015-0011-6

Oakes, L. M. (2012). Advances in eye tracking in infancy research. Infancy 17, 1-8. doi: 10.1111/j.1532-7078.2011.00101.x

Paulus, M. (2014). The emergence of prosocial behavior: why do infants and toddlers help, comfort, and share? Child Dev. Perspect. 8, 77-81. doi: 10.1111/cdep.12066

Rothbart, M. K., Ahadi, S. A., and Evans, D. E. (2000). Temperament and personality: origins and outcomes. J. Pers. Soc. Psychol. 78, 122-135. doi: 10.1037/0022-3514.78.1.122

Salley, B., Miller, A., and Bell, M. A. (2013). Associations between temperament and social responsiveness in young children. Infant Child Dev. 22, 270-288. doi: 10.1002/icd.1785

Schwartz, B., Tesser, A., and Powell, E. (1982). Dominance cues in nonverbal behavior. Soc. Psychol. Q. 45, 114-120. doi: 10.2307/3033934

Shariff, A. F., and Tracy, J. L. (2009). Knowing who's boss: implicit perceptions of status from the nonverbal expression of pride. Emotion 9, 631-639. doi: $10.1037 / \mathrm{a} 0017089$

Shiota, M. N., Campos, B., and Keltner, D. (2003). The faces of positive emotion. Ann. N. Y. Acad. Sci. 1000, 296-299. doi: 10.1196/annals.1280.029

Shum, H. P., Ho, E. S., Jiang, Y., and Takagi, S. (2013). Real-time posture reconstruction for Microsoft Kinect. IEEE Trans. Cybern. 43, 1357-1369. doi: 10.1109/TCYB.2013.2275945

Sirois, S., and Brisson, J. (2014). Pupillometry. Wiley Interdiscip. Rev. Cogn. Sci. 5, 679-692. doi: $10.1002 /$ wcs. 1323 
Sirois, S., and Jackson, I. R. (2012). Pupil dilation and object permanence in infants. Infancy 17, 61-78. doi: 10.1111/j.1532-7078.2011.00096.x

Smith, L., Yu, C., Yoshida, H., and Fausey, C. M. (2014). Contributions of headmounted cameras to studying the visual environments of infants and young children. J. Cogn. Dev. doi: 10.1080/15248372.2014.933430

Steinhauer, S. R., Condray, R., and Kasparek, A. (2000). Cognitive modulation of midbrain function: task-induced reduction of the pupillary light reflex. Int. J. Psychophysiol. 39, 21-30. doi: 10.1016/S0167-8760(00)00119-7

Stommel, M., Beetz, M., and Xu, W. (2015). Model-Free detection, encoding, retrieval, and visualization of human poses from kinect data. IEEE Trans. Mechatron. 20, 865-875. doi: 10.1109/TMECH.2014.2322376

Svetlova, M., Nichols, S. R., and Brownell, C. A. (2010). Toddlers' prosocial behavior: from instrumental to empathic to altruistic helping. Child Dev. 81, 1814-1827. doi: 10.1111/j.1467-8624.2010.01512.x

Tenenbaum, E. J., Shah, R. J., Sobel, D. M., Malle, B. F., and Morgan, J. L. (2013). Increased focus on the mouth among infants in the first year of life: a longitudinal eye-tracking study. Infancy 18, 534-553. doi: 10.1111/j.15327078.2012.00135. $\mathrm{x}$

Tomasello, M., Carpenter, M., Call, J., Behne, T., and Moll, H. (2005). Understanding and sharing intentions: the origins of cultural cognition. Behav. Brain Sci. 28, 675-691. doi: 10.1017/S0140525X05000129

Tracy, J. L., and Robins, R. W. (2004). Show your pride evidence for a discrete emotion expression. Psychol. Sci. 15, 194-197. doi: 10.1111/j.09567976.2004.01503008.x

Tracy, J. L., Robins, R. W., and Lagattuta, K. H. (2005). Can children recognize pride? Emotion 5, 251-257. doi: 10.1037/1528-3542.5.3.251

Troseth, G. L., and DeLoache, J. S. (1998). The medium can obscure the message: young children's understanding of video. Child Dev. 69, 950-965. doi: 10.1111/j.1467-8624.1998.tb06153.x

Walczak, N., Fasching, J., Toczyski, W. D., Morellas, V., Sapiro, G., and Papanikolopoulos, N. (2013). "Locating occupants in preschool classrooms using a multiple RGB-D sensor system," in Proceedings of the 2013 IEEE/RSJ International Conference on Intelligent Robots and Systems (IROS), Tokyo: IEEE, 2166-2172. doi: 10.1109/IROS.2013.6696659
Wang, J. T. Y. (2011). "Pupil dilation and eye tracking," in A Handbook of Process Tracing Methods for Decision Research: A Critical Review and User's Guide, eds M. Schulte-Mecklenbeck, A. Kühberger, and R. Ranyard (New York, NY: Psychology Press), 185-204.

Warga, M., Lüdtke, H., Wilhelm, H., and Wilhelm, B. (2009). How do spontaneous pupillary oscillations in light relate to light intensity? Vision Res. 49, 295-300. doi: 10.1016/j.visres.2008.09.019

Warneken, F. (2015). Precocious prosociality: why do young children help? Child Dev. Perspect. 9, 1-6. doi: 10.1111/cdep.12101

Warneken, F., and Tomasello, M. (2009). Varieties of altruism in children and chimpanzees. Trends Cogn. Sci. 13, 397-402. doi: 10.1016/j.tics.2009. 06.008

Weisfeld, G. E., and Beresford, J. M. (1982). Erectness of posture as an indicator of dominance or success in humans. Motiv. Emot. 6, 113-131. doi: 10.1007/BF00992459

Wilhelm, H. (1991). Pupillenreaktionen - Pupillenstörungen. Stuttgart: Kohlhammer.

Won, A. S., Bailenson, J. N., Stathatos, S. C., and Dai, W. (2014). Automatically detected nonverbal behavior predicts creativity in collaborating dyads. J. Nonverbal Behav. 38, 389-408. doi: 10.1007/s10919-014-0186-0

Zahn-Waxler, C., Radke-Yarrow, M., Wagner, E., and Chapman, M. (1992). Development of concern for others. Dev. Psychol. 28, 126-136. doi: 10.1037/0012-1649.28.1.126

Conflict of Interest Statement: The authors declare that the research was conducted in the absence of any commercial or financial relationships that could be construed as a potential conflict of interest.

Copyright (c) 2015 Hepach, Vaish and Tomasello. This is an open-access article distributed under the terms of the Creative Commons Attribution License (CC BY). The use, distribution or reproduction in other forums is permitted, provided the original author(s) or licensor are credited and that the original publication in this journal is cited, in accordance with accepted academic practice. No use, distribution or reproduction is permitted which does not comply with these terms. 